\title{
JUNO Central Detector and PMT system
}

\author{
Zhimin Wang ${ }^{1}$ \\ Institute of High Energy Physics, CAS \\ 19B, Yuquan Road, Shijingshan District, Beijing, China \\ E-mail: wangzhmeihep.ac.cn \\ Yuguang Xie \\ Institute of High Energy Physics, CAS \\ 19B, Yuquan Road, Shijingshan District, Beijing, China \\ E-mail: ygxiedihep.ac.cn \\ On behalf of JUNO collaboration
}

The Jiangmen Underground Neutrino Observatory (JUNO) is a multi-purpose underground experiment and the largest liquid scintillator (LS) detector going for neutrino mass hierarchy, precise neutrino oscillation parameter measurement and studies of other rare processes which include but not limited to solar neutrino, geo-neutrino, supernova neutrino and the diffuse supernova neutrino background. The $20 \mathrm{kt} \mathrm{LS}$ central detector (CD) of JUNO is the key of the whole facility. Parallel efforts and R\&D activities addressing different aspects of the central detector are being actively pursued at different collaboration institutions. We present here details on the detector design and progresses. In another hand, how to build/install the largest LS detector also is a big challenge for the $\Phi 35.4 \mathrm{~m}$ acrylic sphere vessel with a stainless steel latticed shell and the $\sim 1700020$ " PMT and $\sim 34000$ 3" PMT. Another key design for photon collection and detection is the PMT system, and here also will show a preliminary consideration of the final installation of the whole system including CD, PMT etc. A JUNO detector prototype with $\sim 50 \%$ photon-cathode coverage is under running and analysing. Goals of the prototype include testing different large area PMTs, testing electronics design and detector/data analysis algorithm etc. Preliminary results including PMTs about the prototype will be presented here.

38th International Conference on High Energy Physics

3-10 August 2016

Chicago, USA

\footnotetext{
${ }^{1}$ Dr. Zhimin Wang is born in 1981 and now a research associate in the Institute of High Energy physics of CAS, mainly working on Daya Bay experiment and JUNO experiment in detector, comissioning, calibration, reconstruction and data analysis. 


\section{Introduction}

The Jiangmen Underground Neutrino Observatory (JUNO) [1] will locate at Kaiping of Jiangmen of Guangdong province, south of China, with a $\sim 53 \mathrm{~km}$ baseline to its neighbor reactors as shown in Fig. 1 (left). The first goal of JUNO is going for neutrino mass hierarchy measurement through precisely measured neutrino spectrum as Fig. 1 (right), at the same time, also being a multi-purpose underground experiment and the largest liquid scintillator (LS) detector for precise neutrino oscillation parameter measurement and studies of other rare processes which include but not limited to solar neutrino, geo-neutrino, supernova neutrino and the diffuse supernova neutrino background [2].

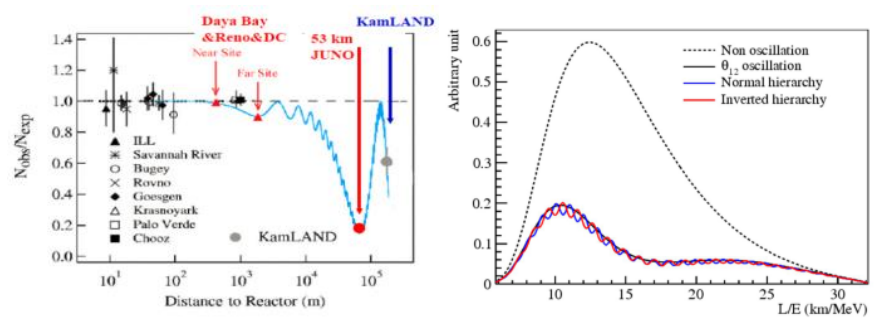

Fig. 1 Left: measured neutrino oscillation versus baseline where JUNO detector will locate at $\sim 53$ km. Right: simulated neutrino energy spectrum for normal (inverted) hierarchy with JUNO.

\section{JUNO detector system}

JUNO is designed with $\sim 20 \mathrm{kt} \mathrm{LS}$ in a $\Phi 35.4 \mathrm{~m}$ acrylic vessel, surounded by at least $4 \mathrm{~m}$ thickness pure water which serves as a shielding layer and an active Muon veto. At the top, there is another additional detector with palstic scintillator layers to provide tracking information of Muons. The rock overburden of the experimental hall is $\sim 700 \mathrm{~m}$. The designed PMT photoncathode cavoerage is $\sim 75 \%$ only with 20 " PMTs.

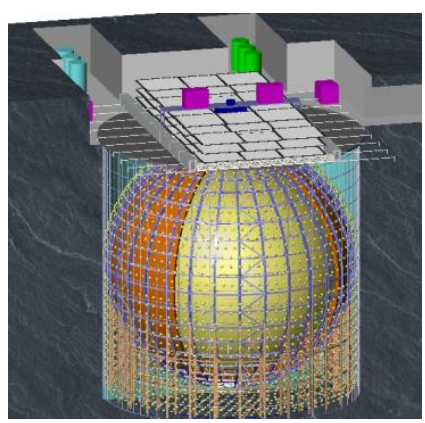

Fig. 2 Overview of JUNO detector system: (from center to edge) LS, sphere acrylic vessel, pure water shielding, stainless steel latticed shell, pure water Muon veto system and top plastic scintillator detector.

\section{$2.1 \quad J U N O$ central detector}

JUNO CD will use $\sim 20 \mathrm{kt} \mathrm{LS}$ as target in sphere acrylic vessel which supported by a stainless steel latticed shell. The acrylic vessel needs to work under gravity force during construction and buoyancy after detector filling. The $\Phi 35.4 \mathrm{~m}$ sphere acrylic vessel with thickness $12 \mathrm{~cm}$ is a real challenge in the world, including sphere plate, bonding, annealing with such huge dimension even without considering presicion, cleaning, safety factors and schedule. Fig. 3 and Fig. 4 show more details and prototypes about 
CD R\&D progresses. Most of the R\&D items are going on well following JUNO schedule.
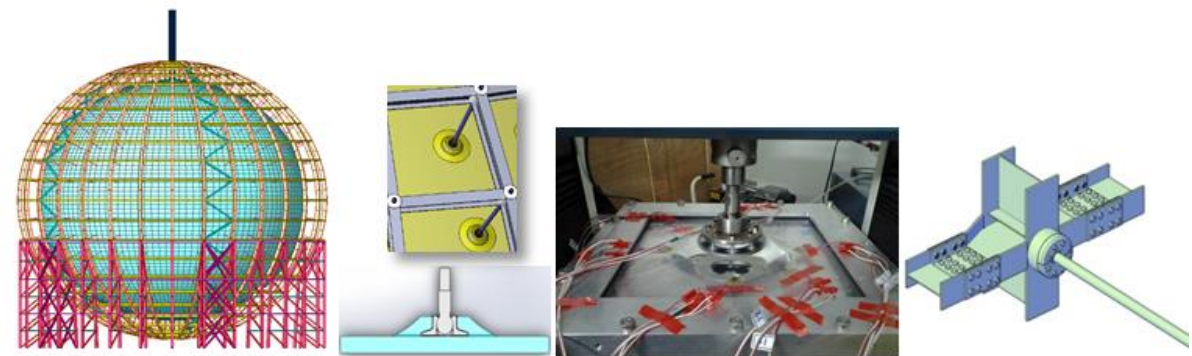

Fig. 3 Left: central detector of JUNO with acrylic vessel and stainless steel latticed shell. Middle left:

Connection between acrylic and support latticed shell and acrylic support node design. Middle right: test of scaled acrylic support node. Right: desgin of stainless steel latticed shell node.

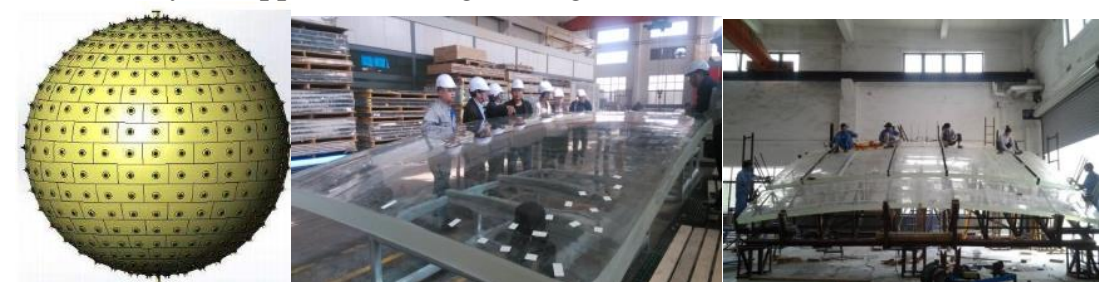

Fig. 4 Left: segmentation of acrylic sphere plates and acrylic support nodes. Middle: prototype for $\Phi 35.4$ $\mathrm{m}$ thickness $12 \mathrm{~cm}$ sphere acrylic plate. Right: bonding prototype with several shaped plates.

\subsection{PMT system}

JUNO detector is designed with $\sim 75 \%$ photon-cathode coverage (Fig. 5) to achieve $\sim 3 \% / \sqrt{ } \mathrm{E}(\mathrm{MeV})$ energy resolution. Totally 15,000 20" NNVT MCP-PMTs and 5,000 20" Hamamatsu PMTs will be used in the detector, at the same time, there also will be at most 34,000 3" PMTs inserted among 20" PMTs as a standalone detection system. The requirements of the two selected 20" PMTs are listed in Fig. 6.
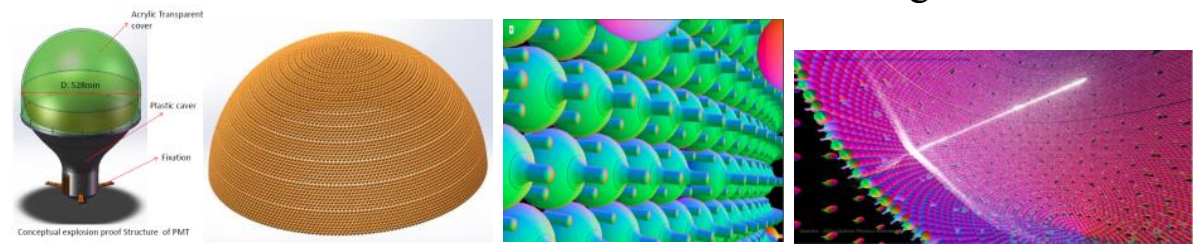

Fig. 5 Left: single 20" PMT module with protection layer. Middle left: PMT distribution on top sphere surface. Middle right: near view at outside of PMT layer, with 3" PMT inserted among 20" PMTs. Right: example of event tracking based on simulation.

Parameters of 20-inch PMT

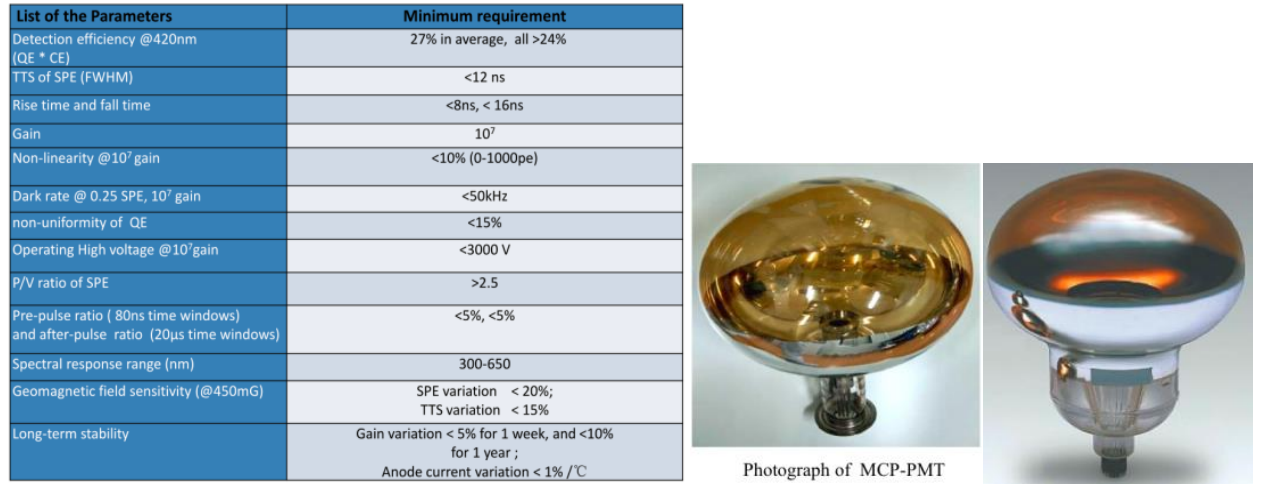

Fig. 6 Left: Requirements of JUNO 20" PMTs. Middle and right: photograph of 20" MCP-PMT and 20" Hamamatsu. 


\subsection{PMT potting and protection}

The PMTs in JUNO will be dipped in pure water with at most $5 \mathrm{~atm}$ pressure. Water proof potting of PMT is one of the keys for system reliability, and a lot of R\&D are going on. The design, testing and production for $1^{\text {st }}$ stage water proof potting were finished well both in signal quality and stability under pressure, where the water proof potting only with PMT+HV divider + single coaxial cable for IHEP JUNO prototype detector (top of Fig. 7). Now, the $2^{\text {nd }}$ round of R\&D with front end electronics (bottom of Fig. 7), which is a challenge for power consumption and temperature control, is going on in material such as waterproof putty, bonding sealants, RTV, cooling, etc. and temperature distribution measurement.

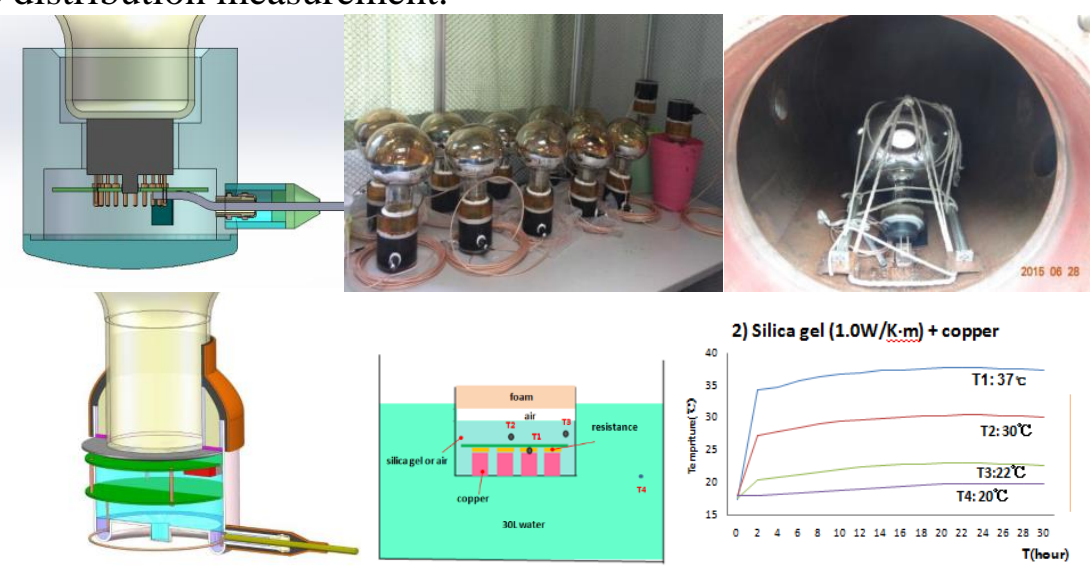

Fig. 7 Top left: the $1^{\text {st }}$ design of water proof potting. Top middle: production of potted 8 " PMTs for JUNO prototype detector. Top right: water pressure test configuration. Bottom left: updated potting structure with electonics in. Bottom middle/right: temperature test configration/results.

The implosion protection of 20" PMTs is developing to avoid the PMTs from chained implosion by any accidents or defect tubes, and the designed structure needs to satisfy such as enough strength to protect the PMTs, good transparency for light, limited dimension by coverage and cost, compatibility with pure water and low radioactivity. The basic idea is to protect the PMT with an additional layer: top is acrylic for transparency and bottom with stainless steel for strength. Till now, many tests have been done with different configurations as shown in Fig. 8. The new designed structure with $12 \mathrm{~mm}$ thickness front acrylic semi-sphere layer is working well in pressed water test. Further optimization is under going.

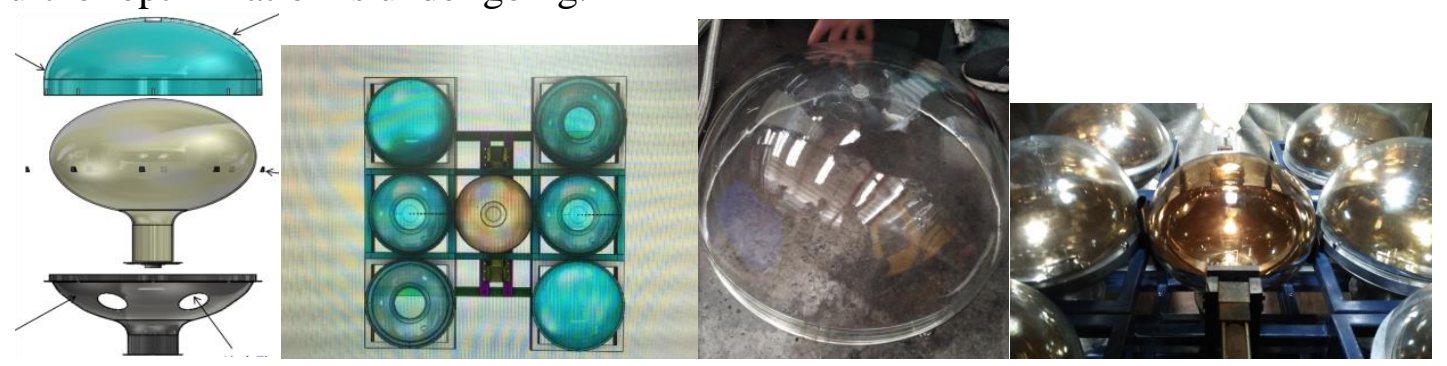

Fig. 8 Left: protection structure. Middle left: drawing of implosion test configuration. Middle right: front acrylic semi-sphere sample. Right: installed implosion test PMTs.

\subsection{PMT installation}

The installation of JUNO PMT system with detector structure is another challenge both views of technics and realization because of limited working space, too many channels, low radioactivity and tight schedule. Several installation options are under 
discussion for all the possibilities with designer and installation companies, and the key strategy which needs to be selected firstly is "Dry" in air or "Wet" in water installation. Fig. 9 is one possible choice, where the stainless steel latticed shell will be installed firstly, and then top acrylic semi-sphere will be installed, at the same time, PMT system will be installed in parallel.

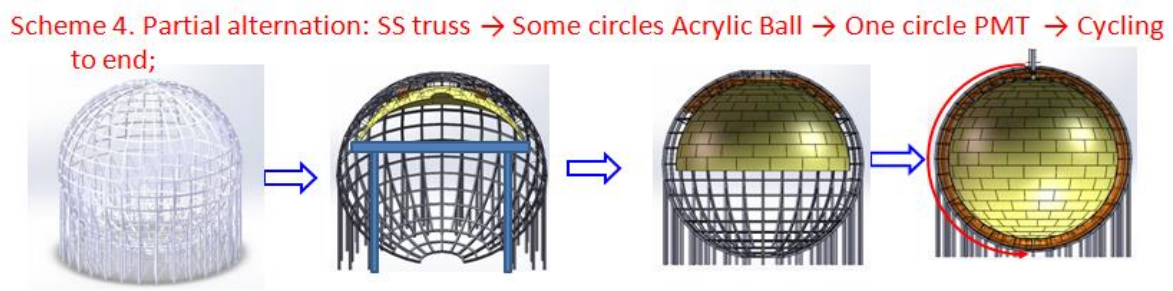

Fig. 9 One example of installation options

\section{Detector Performance}

With the designed detector, JUNO has done a detailed simulation for detector performances as shown in Fig. 10. The detector has a non-uniformity energy response from solid angle effect. With a preliminary vertex reconstruction including PMT waveform, point-like event and tracking event of low and high energy, which vertex resolution can be better than $10 \mathrm{~cm}$ with timing information for point-like event, most of the non-uniformity can be corrected, and the energy relostion will be better than $3 \% / \sqrt{ } \mathrm{E}(\mathrm{MeV})$
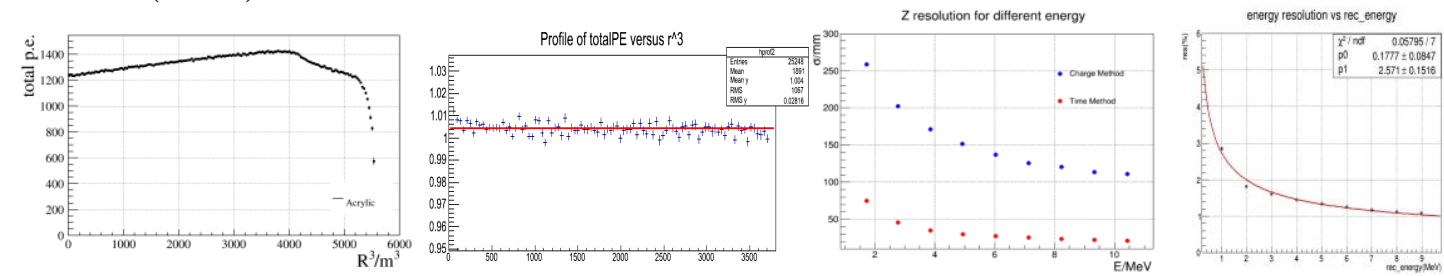

Fig. 10 Left: raw photon-electron number of $1 \mathrm{MeV}$ gamma versus vertex. Middle left: corrected response uniformity. Middle right: reconstructed vertex resolution of point-like with charge or time only information. Right: reconstructed energy resolution of point-like versus energy.

\section{JUNO detector prototype}

In order to study and compare PMTs' performances in a real LS detector, including NNVT MCP-PMT 8" and 20", Hainan Zhanchuang (HZC) PMT 9", and Hamamatsu PMT 8" and 20", a JUNO prototype detector with $601 \mathrm{LS}$ in an acrylic vessel dipped in pure water in a stainless steel tank of $2 \mathrm{~m}$ diameter and height was designed and built at IHEP with similar structure to JUNO and 50\% photon-cathode coverage (Fig. 11). Through the setup of the prototype detector, PMT bench test was done to prepare for PMT mass testing algorithms and cross checked with company parameters. The JUNO prototype detector will get more experiences on new PMT [3], large PMT mounting, large PMT installation, water proof PMT potting, PMT performance in LS detector and detector performance study.
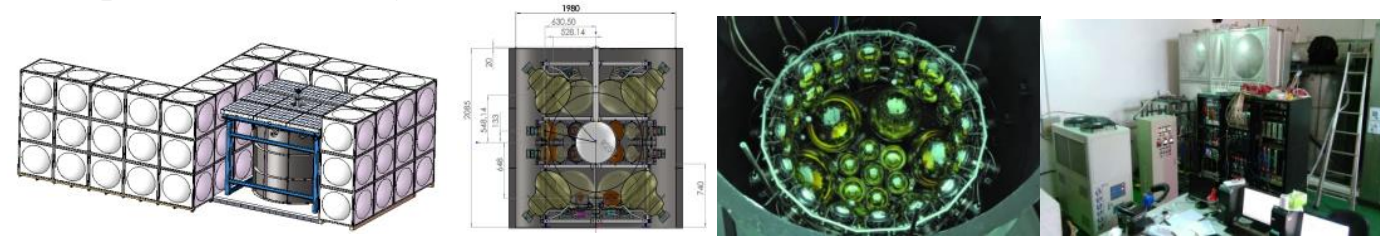

Fig. 11 Left: bird view of the JUNO prototype detector with water shielding tank. Middle left: internal design of the detector including acrylic sphere vessel and PMTs. Middle right: insalled PMTs. Right: installed detector with pure water system, electronics, water tank and detector. 
The prototype detector is installed and doing commissioning, and calibration data were taken. Some preliminary results are very good as shown in Fig. 12. Raw trigger rates is $\sim 300 \mathrm{~Hz}$ with $\sim 0.3 \mathrm{MeV}$ energy threshold, while LS muon rates is $\sim 30 \mathrm{~Hz}$ consistent with expectation (top left of Fig. 12). And the 10 bits $1 \mathrm{GHz}$ waveform sampling electronics works well without dead time for neighbor events to 1 us as configured (top middle of Fig. 12). With gamma and neutron calibration sources, the preliminary reconstructed vertex has a good relationship with its real location to few centimeter (top right of Fig. 12). Bottom left of Fig. 12 shows the neutron capture time on hytrogen in normal LS with AmC neutron source, and bottom middle and right of Fig. 12 are showing the selected prompt and delay signal spectrum where you can see the 2.2 $\mathrm{MeV}$ capture gamma clearly.
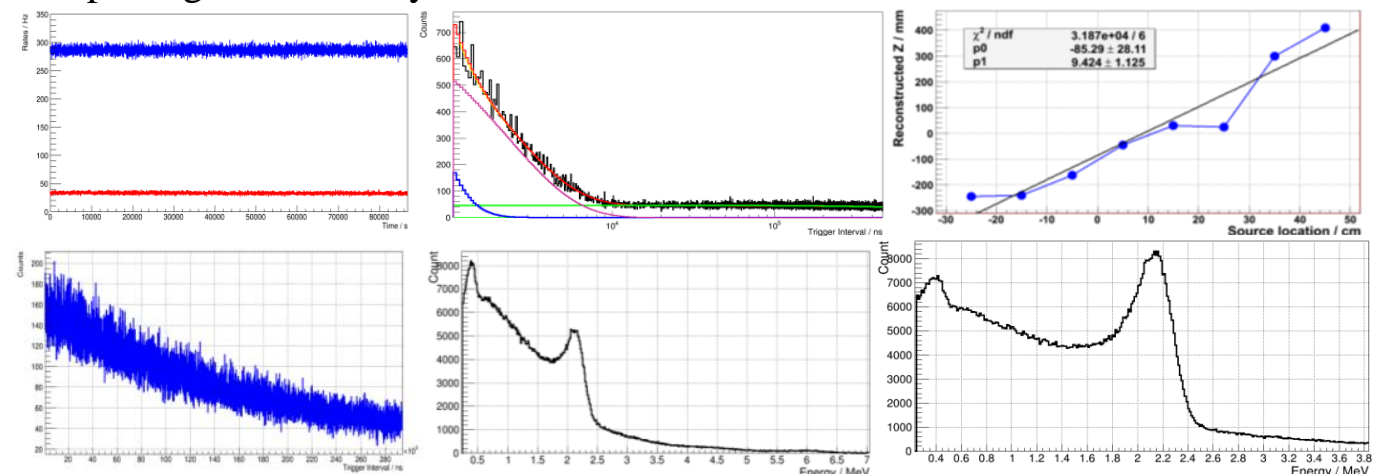

Fig. 12 Top left: raw trigger rates versus time under $\sim 0.3 \mathrm{MeV}$ threshold. Top middle: raw trigger interval of neighbor triggers. Top right: preliminary reconstructed vertex versus real source location. Bottom left: measured neutron capture time spectrum on hytrogen of AmC. Bottom middle (right): selected prompt (delay) signal of AmC source.

\section{Summary}

JUNO detector R\&D are going well according to schedule, including central detector and PMT system. Many key parameters and configurations have been selected. The current schedule of JUNO is to take data at 2020. JUNO prototype detector is constructed and running, preliminary results show a good response of the whole system.

\section{References}

[1] JUNO Conceptual Design Report - JUNO Collaboration (Djurcic, Zelimir et al.) arXiv:1508.07166 [physics.ins-det]

[2] Neutrino Physics with JUNO, JUNO Collaboration (An, Fengpeng et al.) J.Phys. G43 (2016) no.3, 030401 arXiv:1507.05613 [physics.ins-det]

[3] PMT overshoot study for JUNO prototype detector, F.J. Luo etc., CPC accepted, DOI: $10.1088 / 1674-1137 / 40 / 9 / 096002$ 